\title{
The global information society and the implications of self-organization
}

\author{
Frans A.J. Birrer \\ Computer Science \& Society \\ P.O. Box 9512, 2300 RA Leiden, Netherlands \\ Fax: +31715276985 \\ Email: birrer@rulwinw.leidenuniv.nl
}

\begin{abstract}
The emergence of the information society shows many of the characteristics of a self-organizing process. This is certainly true of its currently most publicized branch, the electronic highway. Self-organization, however, implies that it is not possible to steer the process in any direction one might choose; attempts to steer should carefully take into account the social system's own dynamics. A brief analysis of the main imperatives and trends around the electronic highway suggests that we should not be focusing our attention too exclusively on the development of the electronic highway as such. Right now, trends like globalization, decentralization and deregulation are having a much stronger impact. Though information technology (IT) is sometimes called one of the reasons for these trends, they are commonly pursued for quite different reasons. In turn, the trends will shape the future use of IT as well as a lot of other things. If we want the information society to develop in a democratic way, we must face those more general trends.
\end{abstract}

\section{DIAGNOSTICS FOR THE INFORMATION SOCIETY: THE INSTRUMENT OF SOCIOCYBERNETICS}

The information society is developing fast. Structures emerge from numerous initiatives taken in a largely unorchestrated, often even uncoordinated manner. It is hard to get a global picture of what is going on, and what we are heading for. Yet such a general view is exactly what we need, if we want to find out where we stand, and what initiatives are needed. Without it, we run the risk of being caught by the issue of the moment, while missing out on the heart of the matter. 
In such a situation one particularly needs some kind of overall framework to structure the analysis of what is happening. In this paper, I will use a sociocybernetics approach. ' Sociocybernetics is a special branch of the systems paradigm. If one wants to analyze certain complex, interacting phenomena, one can hardly avoid the notions of systems theory. Sociocybernetics is confined to the study of social systems, and takes a specific interest in the phenomena of steering. Steering, here, refers to internal processes that drive the system in a certain direction ('self-steering') as well as attempt to steer the system from the outside. The notion of steering makes it possible to ask more specific questions about the direction of developments, and about the possibilities for intervention.

\section{THE INFORMATION SOCIETY AS A SELF-ORGANIZING SYSTEM}

If there is one currently 'hot' notion from the broad range of systems perspectives that seems particularly applicable to the current developments in the information society, it must be the notion of 'self-organizing system'. The information society does not develop from a preconceived plan, it emerges from the complicated dynamics that result from the initiatives and interplay of many actors exploring new technological possibilities.

There exists no unique definition of the notion of self-organizing system. The term is often used in a rather loose way; as a general term, it functions as a metaphor rather than as a specific model. In the present analysis, the following characteristics of self-organization will be in focus: (i) the structure of the system emerges from a complex interplay of its parts (ii) the dynamics of the system cannot be steered in arbitrary directions (since it depends on the drives and interplay of the parts). I take this to imply the following with respect to what we can and cannot know or predict about such a system:

(1) How existing patterns are kept up by the interplay of various actors is in principle open to analysis. For such an analysis, we must to a certain extent standardize an actor's behaviour, as subjected to more or less predictable 'impulses' (with this term, I am of course not referring to universal drives of behaviour but rather to behaviour as canalized by culture and institutions existing at that particular time and place).

(2) As a first approximation, we can also try to estimate how this existing field of impulses would work out if a new resource, such as a new technology, were introduced. We can analyze what new kinds of opportunities become available to the actors, and the incentives they present.

(3) We cannot - or only to a very limited extent - predict the emergence of entirely new patterns (that is, patterns that do not follow from the two preceding lines of analysis).

(4) We can, to some extent, foresee possible dissonances, that is, consequences of the processes predicted along the lines of (1) and (2) that may not be really wanted by some or all of the actors. We must realize here that we are making a normative anticipation in the name of the actors, which may prove to be wrong. ${ }^{2}$

(5) We cannot predict how actors will actually react to these dissonances. Dissonances may be relieved either by countervailing channels existing in the 
present system, or by changing the procedures along which the system operates. ${ }^{3}$ But discrepancies between outcome and desirable outcome may also lead to (i) new blind impulses (what is called 'technological fix', that is, fighting the unwanted consequences of a certain technology with another one, which again leads to new unwanted consequences, and so on), or (ii) attempts to put the discrepancy out of one's mind (repression, ostrich behaviour). This implies that dissonances can be manifest, but also latent (that is, not formally recognized by the actors).

(6) Finally, the canalization of impulses by culture and institutions as mentioned under (1) is not static. There are shifts in institutional and cultural rules that we must take into account. I use the word 'trends' to denote these shifts (for the topic to be treated here, decentralization, deregulation, and globalization are examples of relevant trends). We can, with some caution, extrapolate such trends for the near future.

\section{ANALYSIS PART I: THE 'BLIND IMPULSES'}

In a self-organizing social system, technology is not a neutral tool, the use of which can be headed in any conceivable direction. As a first approximation, we can see the new technology as a disturbance, inducing the existing blind imperatives to carve new pathways. First order changes can be identified by examining how the technology lowers (or raises) thresholds to perform certain actions. Since the most dramatic changes are currently expected from electronic communication (the Internet, electronic highway, etc.), this will be the main focus. It will be useful to differentiate between specific functions of electronic communication. We can use the following categorization:

\section{Informing}

Impersonal: providing certain information on the net. The information may be accessible by anyone, or only by a selected group of people (for instance, persons who pay for access).

Personal: sending unsolicited information to one or more specific persons. Requesting information

Impersonal: such as by posting a request for information or advice on a list, accessible by others, in the hope that someone is willing to reply.

Personal: consulting a specific person.

\section{Exchanging opinions}

This involves all mutual exchanges with a major evaluative component, which are beyond the informative.

The categories are not completely exclusive (there may not always be a clearcut division between information and opinion), but for the present purpose they are good enough. Since major changes with the Internet lie in new ways of getting together, exchanges that take place in an already existing communicative relation have been left out. 
What kind of structural changes can be expected in each of these areas? In light of what was said earlier about self-organizing systems, this question decomposes into three parts:

(i) In what direction will the natural impulses and imperatives of the social system lead, given the new technological possibilities? This question can be answered by examining what thresholds are lowered compared to the old situation.

(ii) To which types of manifest and latent dissonances might this natural flow of events give rise? Here we must anticipate what consequences might be manifestly or latently disturbing to the actors, as explained before.

(iii) Are there any existing mechanisms through which counterforces could push back disturbing effects?

Let us now briefly pass through the various categories.

\section{Impersonal informing}

Thresholds lowered. Information can be made accessible to a very large public with relatively little effort. Whether the information will actually reach those persons for whom it might be useful is far less obvious. Despite the existence of search engines, the selection problem on the Internet is far from solved.

Potential dissonances. The most obvious nuisance is information overload. Ackoff already suggested that the problem of a manager is not a lack of information, but an excess of it, and the lack of a selection mechanism. ${ }^{4}$ This is equally true of the average Internet user. ${ }^{5}$ If more and more information is provided, the selection problem will become increasingly urgent.

Countermechanisms. Browsers can solve only part of the information overload problem. It is also the way in which information is offered that would have to be guided somehow. Though this may not be impossible in principle, it seems to run counter to the current feel of individual freedom on the Internet. But even if selection were easier, the sheer amount of available information would in many cases still be overwhelming.

\section{Personal informing}

Thresholds lowered. Automated sending of messages can now be done at very low cost. If one has an email address list, pushing one button is enough to send a message to each individual on the list.

Potential dissonances. A main threat here is junkmail. ${ }^{6}$ Since it is so easy to send electronic messages by means of address lists, we might all become flooded with messages; the selection of the relevant ones would become very time consuming, and in extreme cases even the receiving computer system may be overloaded.

Countermechanisms. As far as the source of junkmail can be identified, this problem could be addressed more directly than that of information overload. Insofar as it is possible to send messages where the source cannot be traced, email terrorism might become a real problem. 


\section{Impersonal requests for information}

Thresholds lowered. The net provides instant access to expert communities on an already endless list of topics. Whether one gets a useful response or not depends, of course, on the willingness and capacities of others to answer.

Potential dissonances. I see no imminent dissonances here.

\section{Personal requests for information}

Thresholds lowered. For personal requests for information, the change could be less significant than in the previous cases. Though the current culture on the net seems to lower psychological distances, and seems to make it easier to put a question to someone with whom one has never had contact before, it is also clear that if this were to happen on a large scale, well-known people would probably get a larger number of requests than they could possibly answer.

Potential dissonances. Like in the previous category, there seem to be no major impending dangers here. Getting many requests may be tiresome, but if one simply does not reply any more, the number of requests will probably drop.

\section{Opinion exchange}

Thresholds lowered. There are already discussion lists on almost any topic one could think of. Easy accessibility as well as brief response time have created possibilities that have not been available in any other form before.

Potential dissonances. Some have questioned the usefulness of many Internet exchanges (informative or otherwise). ${ }^{7}$ Some fear a tendency towards superficiality ${ }^{8}$, pseudocommunities ${ }^{9}$ or fragmentation, scattering and alienation ${ }^{10}$. Scanning the interchanges taking place on the Internet may occasionally give rise to feelings of support for such concerns. On the other hand, being patronizing can be dangerous, and it is far from obvious that those interchanges have actually driven out less trivial pursuits.

Countermechanisms. Although the dangers of cultural degradation should not be exaggerated, we must at the same time realize that these effects work behind our backs. We usually are not conscious of them. Because they are so intangible, it is hard to think of effective countermechanisms.

There are a few issues that relate more or less to all of the above categories. Among those are issues that have to do with the (mis)use of information derived from the net. The issue of privacy is one example. Privacy problems, however, are not specific to the net, and they are already addressed by specialized platforms. Registering the entrances at a certain site, for example, for the purposes of profiling or for direct marketing) is another example. The use of IT for direct democracy has similar dangers. Already in 1977, Laudon pointed out that direct democracy by means of the electronic highway could lead to profile research identifying best selling strategies for what has already been decided, rather than result in different decisions or in politicians being more responsive to the wants and needs of citizens. ${ }^{11}$

Finally, dissonances can also arise because some groups or individuals will not be actively involved in the use of electronic communication, and thereby become disadvantaged. Here, schooling is of course the keyword. But in order for 
schooling to be effective, we must be able to present tasks that are motivating and really useful to the ordinary citizen. The inventory above suggests that such tasks may not be immediately available.

On the whole, the main short-term effect seems to be that communication in already existing communicative relations can become more efficient. There may be some threshold lowering for new communicative relations for very specific purposes, but structural short-term change in the information used by citizens is blocked by the phenomenon of information overload.

\section{ANALYSIS PART II: THE 'TRENDS'}

The canalization of impulses that was used to get a first approximation of the direction of developments is of course not invariant. It is itself subject to change. Because changing the rules of the game usually requires discussion, the forces behind such changes are of a cultural nature; they are prone to fashion, and they may even be outright ideological. Shifts in (thinking about) the rules of the game will be called trends. Even if strongly ideological, a trend often contains an idea that makes some sense as long as it is balanced against others; the ideological character lies in the fact that this one aspect is blown up as if there were no other considerations and no trade-offs.

The development of electronic communication is canalized by trends; at the same time, developments in electronic communication may reinforce (or work against) existing general trends. ${ }^{12}$ Some trends that are particularly relevant here are the following:

Decentralization

Deregulation and liberalization

Economic globalization

Cultural globalization

Postmodernism

Each of these trends will now be discussed briefly. In line with the preceding sections, the focus will be on potentially harmful effects of certain trends and, of course, on their interplay with electronic communication.

\section{Decentralization}

There has become an increasing awareness that not all decisions should be made at a central level. Not only would the centre be overburdened, many decisions mainly concern local interests anyway. Since decision- and communication-assisting IT has also developed in a direction of decentralization, the two trends seem to reinforce each other. ${ }^{13}$ It is sometimes suggested that this leads to more democratic control. That, however, very much depends on the way in which the decentralization is carried through. If decentralized processes are strongly standardized, local autonomy may decrease instead of increase. ${ }^{14}$ All this applies to the public sphere as well as private organizations. 


\section{Deregulation and liberalization}

We already saw that in a self-organizing system predictability is limited, and so are the possibilities for steering. The state as implementor of some kind of blueprint master plan for society is not a feasible option. In many western countries there is a trend to reduce the role of the state, and to leave more to deliberation procedures between parties or to market regulation. ${ }^{15}$ Again, however, this withdrawal of the state may reduce the possibilities for a citizen's counterforce. Although the relation between electronic communication and deregulation/ liberalization is more indirect, there is significant coupling through the previously discussed trend of decentralization.

\section{Economic globalization}

The economy is becoming more and more a world economy. Economic decisions in one country are tied to decisions in other countries. Companies can choose where they will conduct their activities, and play off different communities against each other in order to get the most profitable conditions. Modern communication technology is among those factors that make companies more independent of a specific location (some already speak of the virtual organization ${ }^{16}$ ). The result is community autonomy being handed over to firms or to the economic imperatives on which they act. Once more, the result is a serious weakening of potential counteracting forces.

\section{Cultural globalization}

Modern communication technologies have enormously increased people's exposure to cultures other than their own. There is no doubt that this exposure has led to a rather asymmetric adaptation, dominated by western, Anglo-Saxon culture. It is hard to say to what degree this is a bad thing. One may regret that old traditions disappear. Cultural diversity is valued by many as a good in itself. But given our present lifestyle, there are objective reasons for having intensive worldwide communication. Economic globalization makes us interdependent, and there are many other important problems that need to be solved worldwide as well with respect to the environment. To establish adequate deliberations on a world scale, we need a thorough understanding of each other's cultures. If this understanding leads to assimilation, this is not necessarily a bad thing, as long as it is not an involuntary handing over of autonomy. On the other hand, we must realize that the value of a cultural practice is hard to evaluate, and that one might throw something away of which one did not realize the value.

\section{Postmodernism}

Postmodernism is in a sense a philosophical summary of the previous trends. According postmodernism, the time of the big stories (such as political ideologies) is over. There is no unifying framework any more; life and experience are local and fragmented. It is often assumed that this is a good thing for democracy but, again, that is an overhasty conclusion. ${ }^{17}$ Not only are counterforces often most effectively mobilized when the scale is large, they also very much depend on comparisons to other practices as an important argumentative weapon; overemphasizing fragmentation deploys counterforces of an important part of their argumentative 
force. This shows how trends like globalization and decentralization, that apparently go in quite opposite directions, can in fact be seen as two sides of the same coin: it is the counterforces that are fragmented, leaving the field open to the dominant (economic) forces and powers, which then can induce their own uniformities.

Trends like 'deregulation', 'decentralization' and 'globalization' are very powerful at the moment. I put these indications between quotes because, as already indicated, they operate as ideologies; what actually happens under their banner may be very different from what the words suggest. Probably, the trends have selforganizing qualities too. But even though there may be an underlying dynamic that drives the process in a particular direction, it is easier to use canalizing impulses to explain certain structures than to explain changes in the canalization itself, because the drives are much less tangible. For this reason, I have so far treated the trends merely as an exogenous input.

One can, however, suspect at least some of the drives behind the trends. In general, persistent dissonances mostly have to do with load-off effects of some kind. An actor benefits by performing a certain action while others pay the costs. Particularly persistent are situations that present a prisoners' dilemma or a tragedy of the commons (which is in fact a load-off problem and a prisoners' dilemma at the same time). Take information overload. It is due to each individual's wish for personal expression even if, at a collective level, this leads to ineffectiveness. Junkmail is the pursuit of personal interests at the expense of others.

For the potential dissonances arising out of the trends discussed, very similar observations can be made. Deregulation and economic globalization may selectively favour the position of certain parties against that of others. As we saw, certain trends, if pushed too far, will seriously weaken democratic counterforces. Trends like decentralization, deregulation and globalization are attractive to a decision-maker who wants to avoid personal risk: they make it relatively easy to push aside the need for active policy in favour of a construction in which policymakers only acts as facilitators of other people's negotiations and do not have opinions or initiatives of their own. Attractive as this may seem to the decisionmaker, it is severely damaging to the overall problem-solving potential. After all, decision-makers are often installed for the very reason that parties have great difficulty in finding an appropriate solution themselves, because they are involved in a prisoners' dilemma or because the less powerful might be overrun.

Avoidance of personal risk can also be observed in policy initiatives with respect to the information society. ${ }^{18}$ Here too, there is a tendency towards vague rhetoric; there is often insufficient study of what is happening or could realistically happen in the near future and, as a consequence, the resulting programmes do not have sufficiently reflected and concrete goals.

Right now, the impact of the trends seems much stronger than the impact of the electronic highway. Even though these trends are often pictured as unavoidable consequences of the developments of IT, they are not; the drives behind them are of a quite different nature. It is therefore imperative that we do not restrict our view to the electronic highway. The really powerful determinants currently are the ideologies of decentralization, deregulation and globalization. The main direct impact of the projection of electronic utopias will act as an argument to support 
trends that actually are pursued for very different reasons. These ideological policies, in turn, will shape the long-term development of the electronic highway; already, many decision-makers seem interested only in a vigorous commercialization of the net. As a result, publicly important options could be cut off before they even materialize. This will not be stopped unless we find ways to countervail the decision-makers' desire for personal risk avoidance. Like blind impulses, trends also form a self-organizing system. They cannot be changed by direct planning. Our basic task is to eliminate the procedural and rhetorical hiding places that shield biased decisions from external criticism, and to find democratic mechanisms through which countervailing power can be mobilized and effectuated. Maybe we also need a new type of citizen, a citizen who is much more aware of decisions made in various parts of society, and who is willing to influence those decisions more actively. Information technology could support such a project. But then the goals would have to be set very differently from the way they are being set now.

\section{CONCLUSIONS}

It is not the electronic highway, but the trends of 'decentralization', 'deregulation' and 'globalization' that are the most powerful determinants of present developments. We should not be fooled about the true nature and impact of these trends by the rhetoric that accompanies them.

Attempts to influence the development of the electronic highway in the long run will not be successful unless they address these trends, particularly if one is interested in strengthening democracy. Democracy is not an automatic consequence of current IT developments, as is sometimes suggested; on the contrary, if IT is to be used for strengthening democracy, we need a significant change in the present societal structures, providing citizens with real opportunities to influence decisions. We must make sure that decisions of public importance are accounted for in terms of explicit and verifiable arguments. Improvement of the quality of (and participation in) discussions on such decisions is of key importance, and should be stimulated by education of the public as well as of decision-makers.

With respect to the electronic highway this implies at least the following: (i) citizens must have access to the net at a price that is affordable for everyone, (ii) all information that has public relevance should be freely available on the net, in a form that is as understandable and as transparent as possible to the general public, and (iii) citizens should be enabled with real possibilities to influence decisions.

We must beware of technocracy and utopian schemes. Assuming that everything operates ideally is unrealistic and dangerous. Social systems cannot be steered in any way we choose. We must be prepared to formulate concrete diagnoses, and to make specific, well-considered interventions.

\section{ACKNOWLEDGEMENTS}

I would like to thank the members of the Dutch study group for sociocybernetics and the participants at the conference for their comments. 


\section{REFERENCES}

Ackoff Russell L., Management Misinformation Systems, Management Science, 14, no. 4, B-147-156 (December 1967)

Beniger James, The control revolution, 1986, Harvard University Press, Cambridge (MA)

Berghel Hal, Cyberspace 2000: dealing with information overload, Communications of the ACM, 40, no. 2 (February 1997) 19-24

Besser Howard, From Internet to Information Superhighway, in: James Brook, Iain A. Boal (eds.), Resisting the virtual life. The culture and politics of information, 1995, City Lights, San Francisco

Birrer Frans A.J., Expertise, context and problem orientation. A constructive realism perspective on science and expertise, 1994, Technical Report 94-45, Dept. of Computer Science, Leiden University

Birrer Frans A.J., Client-oriented anticipation in expert-advised problem solving, 1996, Technical Report 96-40, Dept. of Computer Science, Leiden University

Bryant Tony, The myth of the information society, in: Jacques Berleur, John Drumm (eds.), Information technology assessment (Proceedings 4th Human Choice and Computers Conference), 1991, North-Holland, Amsterdam

Danziger James N., William H. Dutton, Rob Kling, Kenneth L. Kraemer, Computers and politics. High technology in American local government, 1982, Columbia University Press, New York

Dutton William, The political implications of information technology: Challenge to power?, in: Jacques Berleur, Andrew Clement, Richard Sizer, Diane Whitehouse (eds.), The information society. Evolving landscapes, 1990, Springer, New York

European Commission, Green paper. Living and working in the information society: People first, 1996

Frissen P.H.A., De virtuele staat. Politiek bestuur, technologie: een postmodern verhaal, 1996, Academic Service, Schoonhoven

Geyer Felix, \& van der Zouwen J., Cybernetics and social science. Theories and research in sociocybernetics, Kybernetes, 20, no. 6, December 1991

Information Society Forum, Networks for people and their communities. Making the most of the information society in the European Union (Report to the European Commission), 1996

Laudon Kenneth C., Communications technology and democratic participation, 1977, Praeger, New York

Miller Steven E., Civilizing cyberspace. Policy, power, and the information superhighway, 1996, ACM Press, New York

Abbe Mowshowitz, Virtual feudalism: a vision of political organization in the information age, in: P.H.J. Frissen, A.W. Koers, I. Snellen (eds.), Orwell of Athene? Democratie en informatiesamenleving, 1992, SDU, The Hague

Qvortrup Lars, The information age. Ideal and reality, in: Jennifer Daryl Slack, Fred Fejes (eds.), The ideology of the information age, 1987, Ablex, Norwood (NJ)

Robins Kevin, Webster Frank, Information as capital. A critique of Daniel Bell, in: Jennifer Daryl Slack, Fred Fejes (eds.), The ideology of the information age, 1987, Ablex, Norwood (NJ)

Schiller Herbert I., Communication of knowledge in an information society, in: Jacques Berleur, Andrew Clement, Richard Sizer, Diane Whitehouse (eds.), The information society. Evolving landscapes, 1990, Springer, New York

Stoll Clifford, Silicon snake oil. Second thoughts on the information highway, 1995, Doubleday, New York

Talbott Stephen L., The future does not compute. Transcending the machines in our midst, 1995, O'Reilly, Sebastopol (CA)

Zuboff Shoshana, In the age of the smart machine. The future of work and power, 1988, Basic, New York.

For an overview of sociocybernetics, see [Geyer \& Van der Zouwen, 1991].

2 I have argued elsewhere [Birrer, 1994; 1996] that such normative anticipation on behalf of actors is quite a common phenomenon in science and scientific expertise, even though it is not often acknowledged.

3 This again follows from the limited possibilities for active steering in a self-organizing system. The behaviour of the system cannot be adjusted in arbitrary ways or directions; any attempt to 
steer should take into account the imperatives that are at work in the system. In order to get different overall outcomes one has to change the procedures of the system itself, so that the blind imperatives are canalized in a different way.

4 [Ackoff, 1967].

5 See, for example, [Berghel, 1997].

6 See, for example, [Miller, 1996, p. 272-274].

$7 \quad$ [Stoll, 1995].

8 [Besser, 1995].

$9 \quad C f$. [Beniger, 1986].

10 [Talbott, 1995].

11 [Laudon, 1977].

12 That has often been the role of IT, see, for example, [Beniger, 1986], [Bryant, 1991], [Danziger, et al., 1982], [Dutton, 1990], [Qvotrup, 1987], [Robins \& Webster, 1987], and [Schiller, 1990]

3 [Frissen, 1996].

14 Cf. [Beniger, 1986], [Zuboff, 1988].

15 Cf. Miller's description of an economic tragedy of the commons [Miller, 1996, p.387-80].

16 [Mowshowitz, 1992].

17 Cf. [Talbott, 1995].

18 For example, [European Commission, 1996], [Information Society Forum, 1996]. 